\title{
Stochastic Traffic Engineering in Multihop Cognitive Wireless Mesh Networks
}

\author{
Yang Song, Student Member, IEEE, Chi Zhang, and Yuguang Fang, Fellow, IEEE
}

\begin{abstract}
In this work, the stochastic traffic engineering problem in multihop cognitive wireless mesh networks is addressed. The challenges induced by the random behaviors of the primary users are investigated in a stochastic network utility maximization framework. For the convex stochastic traffic engineering problem, we propose a fully distributed algorithmic solution which provably converges to the global optimum with probability one. We next extend our framework to the cognitive wireless mesh networks with nonconvex utility functions, where a decentralized algorithmic solution, based on learning automata techniques, is proposed. We show that the decentralized solution converges to the global optimum solution asymptotically.
\end{abstract}

Index Terms—Cognitive networks, network utility maximization, learning algorithms.

\section{INTRODUCTION}

$\mathrm{T}$ HE past decade has witnessed the emergence of new wireless services in daily life. One of the promising techniques is the metropolitan wireless mesh networks $(\mathrm{WMN})$, which are envisioned as a technology which advances toward the goal of ubiquitous network connection. Fig. 1 illustrates an example of wireless mesh network. The wireless mesh network consists of edge routers, intermediate relay routers as well as the gateway node. Edge routers are the access points which provide the network access for the clients. The relay routers deliver the traffic aggregated at the edge routers to the gateway node, which is connected to the Internet, in a multihop fashion.

While the current deployed wireless mesh networks provide flexible and convenient services to the clients, the performance of a mesh network is still constrained by several limitations. The first barrier is due to the multihop nature of the wireless mesh network, where the nodes in geographic proximity generate severe mutual interference among each other and thus the network performance is devastated. To address this problem, several scheduling schemes have been proposed in the literature [1]. Recently, a novel coding-based scheme which may produce an interference-free wireless mesh network, is proposed [2]. Another example of the interference-free network is the CDMA-based wireless mesh networks [3] where by assigning orthogonal codes for each link, the network throughput is remarkably improved.

The second hindrance for the network performance is the limited usable frequency resource. In current wireless mesh

- Y. Song and C. Zhang are with the Department of Electrical and Computer Engineering, Engineering Building, University of Florida, Gainesville, FL 32611. E-mail: \{yangsong, zhangchi\}@ufl.edu.

- Y. Fang is with the Department of Electrical and Computer Engineering, Engineering Building, University of Florida, Gainesville, FL 32611 and is also a Changjiang Scholar Chair Professor with the National Key Laboratory of Integrated Services Networks, Xidian University, Xi'an 710071, China. E-mail: fang@ece.ufl.edu.

Manuscript received 8 Jan. 2009; revised 30 Apr. 2009; accepted 26 May 2009; published online 2 June 2009.

For information on obtaining reprints of this article, please send e-mail to: tmc@computer.org, and reference IEEECS Log Number TMC-2009-01-0011. Digital Object Identifier no. 10.1109/TMC.2009.111.

$1536-1233 / 10 / \$ 26.00$ (C) 2010 IEEE networks, the unlicensed ISM bands are most commonly adopted for backbone communications. Not surprisingly, the wireless mesh network is largely affected by all other devices in this ISM band, e.g., nearby WLANs and Bluetooth devices. Moreover, the limited bandwidth of the unlicensed band cannot satisfy the increasing demand for the bandwidth due to the evolving network applications. Ironically, as shown by a variety of empirical studies [4], the current allocated spectrum is drastically underutilized. As a consequence, the urge to explore the unused white space of the spectrum, which can significantly enhance the performance of the wireless mesh networks, attracts tremendous attention in the community [5], [6], [7], [8], [9].

Cognitive radios are proposed as a viable solution to the frequency reuse problem [1]. The cognitive devices are capable of sensing the environment and adjusting the configuration parameters automatically. If the primary user, i.e., the legitimate user, is not using the primary band currently, the cognitive devices, namely, secondary users, will utilize this white space of the spectrum. Incorporating with the established interference-free techniques such as [2] and [10], the throughput of the wireless mesh network can be dramatically enhanced. The protocol design for cognitive wireless mesh networks (CWMN), or more generally, multihop cognitive radio networks, is an innovative and promising topic in the community [11] and has been less studied in the literature. In this paper, we consider a cognitive wireless mesh network where the unlicensed band, e.g., ISM band, is utilized by the mesh routers for the backbone transmission. Moreover, each router is a cognitive device and hence is capable of sensing and exploiting the unused primary bands for transmissions whenever the primary users are absent.

In this paper, we investigate an important yet unexplored issue in the cognitive wireless mesh networks, namely, the stochastic traffic engineering (STE) problem. More specifically, we are particularly interested in how the traffic in the multihop cognitive radio networks should be steered, under the influence of random behaviors of primary users. It is worth noting that given a routing 


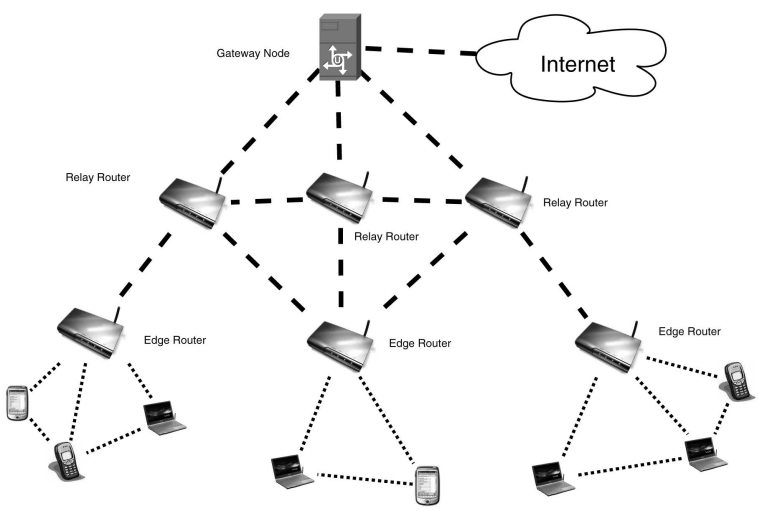

Fig. 1. Architecture of wireless mesh networks.

strategy, the corresponding network's performance, e.g., the average queuing delay encountered, is a random variable. The reason is that the available bandwidth for a particular link depends on the appearance of all the affecting primary users. If all the primary users are vacant, a link can utilize all available frequency trunks collectively by utilizing advanced physical layer techniques, e.g., OFDMA. However, if all the primary users are present, the only available frequency space is the unlicensed ISM band and thus the traffic on this link will experience longer delay than the previous case. In other words, the performance of a traffic engineering solution hinges intensely on the unpredictable random behaviors of the primary users. We emphasize that in multihop cognitive radio networks, this distinguishing feature of randomness, induced by the random behaviors of primary users, must be taken into account in protocol designs. Due to the location discrepancy, it is possible that some node is affected by many primary users while others are not. As a consequence, if we route the traffic via this particular node, the transmissions are more likely to be corrupted by the returns of the primary users. Apparently, a favorable solution is more inclined to steer the traffic from those "severely affected area," to the paths which are less affected by the primary users. We will make this intuitive approach precise and rigorous in this paper. To our best knowledge, this paper is the first work on the traffic engineering problem in multihop cognitive radio networks, with a special focus on the impact of random behaviors from the primary users.

The rest of this paper is organized as follows: The related work is reviewed in Section 2, and Section 3 provides the system model of our work. The stochastic traffic engineering problem with convexity is investigated in Section 4. In Section 5, we extend our framework to the nonconvex stochastic traffic engineering problem. Performance evaluation is provided in Section 6, followed by concluding remarks in Section 7.

\section{Related Work}

Traditional traffic engineering (TE) algorithms are proposed as the solution to the traffic management of the network in a cost-efficient manner. Different from the traditional quality of service (QoS) routing, the traffic engineering solution not only guarantees a certain QoS level for each flow, but also optimizes a global performance metric over the whole network, by splitting the ingress traffic optimally among several available paths. The multipath routing is usually supported by the Multiprotocol Label Switching (MPLS) techniques where the explicit routing path for a packet is predetermined rather than being computed in a hop-by-hop fashion. For a pair of source and destination nodes, the set of available paths, a.k.a., label switched paths (LSP), are established and managed by signaling protocols such as RSVP-TE [12] and CR-LDP [13] or manual configuration. The traditional traffic engineering solution evolves to the STE solution when uncertainty exists in the network, e.g., the random returns of the primary users in our scenario. TE solutions require consistent route changes which are unfavorable in that the network will be overwhelmed by the oscillations induced by the unpredictable behaviors of the primary users. In light of this stability concern, STE solution alternatively pursues an optimum multipath routing strategy such that the expected utility of the network is maximized. The stochastic traffic engineering with uncertainties are discussed in the literature such as in [14] and [15]. However, the previous works usually assume a probability distribution of the uncertainty, while in our scenario, the behaviors of the primary users are completely unpredictable from the mesh network's point of view. Distinguishing from the previous works, we propose an algorithmic solution which requires no prior knowledge about the distribution of the uncertainty, in a stochastic network utility maximization framework. It is worth noting that our work differs from the traditional state-dependent traffic engineering solution as well. For example, in [16], a state-dependent traffic engineering solution is proposed. However, the authors assume that the system state, i.e., the current value of uncertainty, is fully observable. In our approach, we do not assume that the ingress node has the perfect knowledge of the current appearance of the network. We will discuss this issue in detail in Section 4.

Recently, CWMN have attracted great attention in the literature. In [5], the channel assignment is discussed in a CWMN. In [6], a cluster-based cognitive wireless mesh network framework is proposed. The infrastructure-based cognitive network is discussed in [7] with a focus on the cooperative mobility and the channel selection schemes. The spectrum sensing and channel selection are jointly considered in a unified framework in [8]. In addition, the IEEE $802.16 \mathrm{~h}$ is in the process of incorporating the cognitive radios into the WiMAX mesh networks [9]. However, none of the previous works considers the stochastic traffic engineering problem. Therefore, a systematic study of the impacts of the random returns from the primary users, on the network routing performance is lacking in the existing literature. In [17], the joint congestion control and traffic engineering problem is considered. He et al. propose a distributed algorithm to balance the user's utility and the system's objective. However, the authors assume the environment is fixed and does not consider the randomness which is the distinguishing yet usually overlooked feature in cognitive radio networks. Wang and Zheng [18] and Xin et al. [19] discuss the routing issue in cognitive radio networks yet the impact of random returns of primary users is not investigated. Hou et al. [20], [21], [22], [23] formulate the joint routing, power and subband allocation problem in 
cognitive radio networks as a mixed-integer programming. However, the channels' bandwidths are assumed to be fixed, i.e., the random behaviors of primary users are still neglected. Our work is partially inspired by Lee et al. [24]. However, our paper differs from theirs in three crucial aspects. First, by targeting the stochastic traffic engineering problem, our model differs from the joint power scheduling and rate control work in [25]. Second, in [24], [25], Lee et al. only consider a single-path scenario while our work extends to a multipath routing network where the network traffic can be steered. Third and most importantly, Lee et al. [24], [25] require that the current system state is fully observable at the decision maker. To achieve this, the authors assume a centralized mechanism which knows all the channel states of all the links over the network. However, our work differs from [24], [25] significantly in that we do not require that the current system's state is known, which is of great practical interest since in multihop cognitive wireless mesh networks, the decision makers, i.e., the edge routers in our scenario, cannot be aware of the appearance of all primary users in the whole network as a priori. Moreover, our schemes enjoy a decentralized implementation, in contrast to centralized mechanisms in [24], [25] by utilizing the feedback signals and local information only. In our previous work of [26], we proposed a routing optimization scheme to combat with the randomness of instantaneous traffic in noncognitive wireless mesh networks. With respect to [26], this paper differs in the following ways: First, in the wireless mesh networks considered in [26], the capacity of each wireless link is assumed to be fixed, i.e., time invariant. However, in cognitive wireless mesh networks, due to the unpredictable appearance of primary users, the bandwidth of each wireless link is random. Second, in [26], the QoS requirement is not considered. Nevertheless, in this paper, we particularly address the QoS concern of each user, e.g., the expected accumulated delay on the paths cannot exceed a user-specific delay tolerance, as will be elaborated in Section 4. Third and most importantly, the analysis in [26] was based upon the assumption that all the users have convex utility functions. In this paper, we extend the techniques to address the scenarios with nonconvex utility functions. We will discuss the aforementioned issues further in the following sections.

\section{System Model}

We consider a multihop wireless mesh network illustrated in Fig. 1 where an uplink traffic model is considered, i.e., all edge routers aggregate the traffic from clients and deliver to the gateway node via the intermediate relay routers. To ensure connectivity, we utilize the ISM $2.4 \mathrm{G}$ band as the underlying common channel for the wireless mesh network. In addition, each link can utilize the opportunistic channels, i.e., secondary bands to increase the link's achievable data rate whenever the primary user is vacant. We assume that there exists ${ }^{1}|\mathbb{M}|$ primary users. Each primary user possesses a licensed frequency channel and each mesh router is a cognitive node which has the capability of sensing the current wireless environment. We model the multihop cognitive wireless mesh network as a directional graph $\mathcal{G}$ where the vertices are the nodes. We

1. The symbol of $|\mathbb{X}|$ represents the cardinality of the set $\mathbb{X}$. also denote link $(i, j)$ as link $e, e \in \mathbb{E}$ where $t(e)=i$ and $r(e)=j$ represent the transmitter and the receiver of link $e$.

We first consider a particular link denoted by $(m, n)$. The instantaneous available frequency bands, at time $t$, for a node $i$ is denoted by $I_{i}(t)$, which is determined by the current presence of the primary users. Besides the underlying ISM band, the communication between $m$ and $n$ can further utilize all secondary bands within $I_{m}(t) \bigcap I_{n}(t)$, if available. The current cognitive radio devices benefit largely from the software-defined radio (SDR) techniques with advanced coding/modulation capabilities. For example, by utilizing the multicarrier modulation, e.g., OFDMA, a cognitive radio device can utilize all the disjoint available frequency band simultaneously [20], [21], [27], [28], [29]. At the transmitter, a software-based radio combines waveforms for different subbands and thus transmit signal at these subbands simultaneously. While at the receiver, a software-based radio decomposes the combined waveforms and thus receives signal at these subbands simultaneously [22], [23], [28], [29]. In this paper, we assume a spectrum sensing scheme available that each node can sense the presence of the primary users in range, such as [1], [30], although the time of random returns cannot be predicted. A link will utilize all the available vacant bands and that the cognitive radios are full-duplex and can transmit at different bands concurrently [22], [23], [28], [29]. We further assume that some scheduling mechanism is in place or some physical layer mechanisms are utilized such that the nodes cannot interfere with each other during the transmissions. For example, in a multichannel multiradio wireless mesh network, the channels can be assigned properly that the transmissions do not interfere with the neighboring nodes [2], [31]. Other examples are the OFDMA/CDMA-based wireless mesh networks [3], [32] where the interference among nodes can be eliminated by assigning orthogonal subcarriers/codes. We emphasize that this assumption is only for the sake of modeling simplicity and does not incur any loss of generality, as will be clarified shortly.

It is worth noting that the available bandwidth of each link in the cognitive wireless mesh network is a random variable. For example, at time instance $t_{1}$, node $m$ has three secondary bands available, i.e., $I_{m}\left(t_{1}\right)=\left\{I_{0}, I_{1}, I_{2}, I_{3}\right\}$ and $I_{n}\left(t_{1}\right)=\left\{I_{0}, I_{2}, I_{3}, I_{4}, I_{5}\right\}$ due to the location discrepancy, where band 0 is the underlying unlicensed ISM band and $1,2,3,4,5$ are the licensed bands of primary users. The current bandwidth of link $(m, n)$ is represented by $W_{m, n}\left(t_{1}\right)=B W_{0}+B W_{2}+B W_{3}$ where $B W_{i}$ is the bandwidth of band $i$. At another time instance $t_{2}$, the primary user 2 returns and the bandwidth of link $(m, n)$ becomes $W_{m, n}\left(t_{2}\right)=B W_{0}+B W_{3}$. In other words, the bandwidth of links are random variables which are determined by the unpredictable appearance of the primary users. We model this randomness induced by the primary users as a stationary random process with arbitrary distribution. The system is assumed to be time slotted. In each time slot $n$, the system state is assumed to be independent and is denoted by a state vector $s=\left\{\delta_{1}, \ldots, \delta_{|\mathbb{M}|}\right\}, s \in \mathbb{S}$, where $\delta_{i}=1$ denotes the absence of the $i$ th primary user and 0 otherwise. We denote the stationary probability distribution of state $s$ 
as $\pi_{s}$. For the ease of exposition, we assume that the primary users are static. However, we emphasize that our model can be extended to mobile primary users scenarios straightforwardly. For example, if a primary user is moving following a Markovian walk model with well-defined steady-state distribution, the following analysis still applies. Without loss of generality, we express the link capacity in the form of CDMA-based networks, i.e., the capacity of a wireless link $e \in \mathbb{E}$, given the system state $s$, is denoted by $c_{e}^{s}$, which is given by Chiang [33] and Chiang et al. [34] $c_{e}^{s}=W_{e}^{s} \frac{1}{T} \log _{2}\left(1+K \gamma_{e}^{s}\right)$, where $W_{e}^{s}$ is the bandwidth of link $e$ in state $s$ and $\gamma_{e}^{s}$ is the current SINR value of link $e$. The constant $T$ is the symbol period and will be assumed to be one unit without loss of generality [34]. The constant $K=$ $\frac{-\Phi_{1}}{\log \left(\Phi_{2} B E R\right)}$ where $\Phi_{1}$ and $\Phi_{2}$ are constants depending on the modulation scheme and $B E R$ denotes the bit error rate. We will assume $K=1$ in this paper for simplicity [33]. Note that our network model can be incorporated into other types of networks such as MIMO, OFDM with TDMA, or CSMA/CA-based MAC protocols by modifying the form of the capacity accordingly, which represents the achievable data rate in general. For example, if we consider a scheduling-based MAC protocol where each link obtains a time share of the channel access, the achievable data rate is given by $c_{e}^{s}=\widetilde{c_{e}^{s}} \times \psi_{e}$ where $\psi_{e}$ is the fraction of time that the link is active following the scheduling scheme and $\widetilde{c_{e}^{s}}$ is the nominal Shannon capacity of the link.

There are $|\mathbb{L}|$ unicast sessions in the network, denoted by set $\mathbb{L}$, where each session $l$ has a traffic demand $d_{l}$. We associate each session with a unique user. Therefore, we will use session $l$ and $u s e r ~ l$ interchangeably. For each session $l \in \mathbb{L}$, we denote the source node and destination node as $S(l)$ and $D(l)$, respectively. Recall that we assume an uplink traffic model and thus all the source nodes are edge routers and the destination node is the gateway. Furthermore, to improve the reliability and dependability, we allow multipath routing schemes. We denote the available ${ }^{2}$ set of acyclic paths from $S(l)$ to $D(l)$ by $\mathbb{P}_{l}$ and the $k$ th path is represented by $P_{l}^{k}$. We introduce a parameter $r_{l}^{k}$ as the flow allocated in the $k$ th path of session $l$. The overall flow of user $l$, represented by $x_{l}$, is given as

$$
x_{l}=\left[\sum_{k=1}^{\left|\mathbb{P}_{l}\right|} r_{l}^{k}\right]_{0}^{d_{l}},
$$

where $[x]_{a}^{b}$ denotes $\max \{\min \{b, x\}, a\}$. Define an $|\mathbb{E}|$-by- $\left|\mathbb{P}_{l}\right|$ matrix $\mathbb{H}_{l}$ where the element $H_{e, k}^{l}=1$ if link $e$ is on the $k$ th path of $\mathbb{P}_{l}$ and 0 otherwise. Hence, $\mathbb{\mathbb { I }}=\left\{\mathbb{H}_{1}, \ldots, \mathbb{I I}_{|\mathrm{L}|}\right\}$ represents the network topology. Note that the traffic splitting and the source routing are executed on the source node $S(l)$.

For each link $e \in \mathbb{E}$, there is an associated cost function, denoted by $l_{e}^{s}\left(f_{e}, c_{e}^{s}\right)$ where $f_{e}$ is the accumulated flow on link $e$. We assume the function $l_{e}^{s}$ is an increasing,

2. The available set of multiple paths can be obtained by signaling mechanisms such as RSVP-TE [12] or preconfigured manually. In this paper, we assume a predetermined set of acyclic paths. The protocol design for acquiring such paths is beyond the scope of this paper. differentiable, and convex function of $f_{e}$ for a fixed $c_{e}^{s}$. For example, if we assume $l_{e}^{s}\left(f_{e}, c_{e}^{s}\right)=\frac{1}{c_{e}^{s}-f_{e}}$ when $c_{e}^{s} \geq f_{e}$, the cost essentially represents the delay for a unit flow on link $e$ under the $M / M / 1$ assumption. Note that in our scenario, even the accumulated flow $f_{e}$ is fixed, the value of cost function is random due to the state-dependent variable $c_{e}^{s}$. From the network's perspective, the stochastic traffic engineering solution will distribute the aggregated flow among multiple paths optimally, in the sense that the overall network utility is maximized. In next section, we will formulate the stochastic traffic engineering problem in a stochastic network utility maximization framework [34] and provide a distributed solution which requires no prior information about the underlying probability distribution, i.e., $\pi_{s}$, of the system states.

\section{Stochastic Traffic Engineering With CONVEXITY}

\subsection{Formulation}

In the standard network utility maximization framework, each user has a utility function $U_{l}\left(x_{l}\right)$, which reflects the degree of satisfaction of user $l$ by transmitting at a rate of $x_{l}$, e.g., $U_{l}\left(x_{l}\right)=\log \left(x_{l}\right)$. In this section, we assume the utility functions to be concave and differentiable. The nonconvex utility functions are considered in Section 5. Note that the fairness issue can be embodied in the utility functions [34]. For example, in the seminal paper [35], the log-utility functions are adopted to achieve the proportional fairness among different flows.

Define a feasible stochastic traffic engineering solution as $\mathbf{r}=\left[\mathbf{r}_{1}, \ldots, \mathbf{r}_{|\mathbb{L}|}\right]$ where $\mathbf{r}_{l} \triangleq\left[r_{l}^{1}, \ldots, r_{l}^{\left|\mathbb{P}_{l}\right|}\right]$. We can formulate the stochastic traffic engineering problem as $\underline{\mathcal{P}_{1}:}$

$$
\begin{gathered}
\max _{\mathbf{r} \geq \mathbf{0}} \sum_{l \in \mathbb{L}} U_{l}\left(\sum_{k \in \mathbb{P}_{l}} r_{l}^{k}\right) \\
\text { s.t. } \\
\sum_{k \in \mathbb{P}_{l}} r_{l}^{k} \leq d_{l} \forall l \in \mathbb{L}, \\
\sum_{s \in \mathbb{S}} \pi_{s} \sum_{k \in \mathbb{P}_{l}} r_{l}^{k}\left(\sum_{e \in P_{l}^{k}} l_{e}^{s}\left(f_{e}, c_{e}^{s}\right)\right) \leq b_{l} \forall l \in \mathbb{L}, \\
f_{e} \leq \sum_{s \in \mathbb{S}^{\prime}} \pi_{s} c_{e}^{s} \forall e \in \mathbb{E}, \\
f_{e}=\sum_{l \in \mathbb{L}} \sum_{k \in \mathbb{P}_{l}} H_{e, k}^{l} r_{l}^{k} \forall e \in \mathbb{E}, \\
c_{e}^{s}=W_{e}^{s} \frac{1}{T} \log _{2}\left(1+K \gamma_{e}^{s}\right) \forall e \in \mathbb{E},
\end{gathered}
$$

where $e \in P_{l}^{k}$ represents the links along the $k$ th path of user $l$. The variable in $\mathcal{P}_{1}$ is the vector of $\mathbf{r}$. The first set of constraints reflect that the overall data rates of all paths 
cannot exceed the traffic demand $d_{l}$. The second set of constraints indicate that for each user $l$, the expected cost has to be no more than a predefined constraint $b_{l}$. The third set of constraints represent that the aggregated flow on link $e$ cannot exceed the average link capacity. Apparently, if the underlying probability distribution of each state $\pi_{s}$ is known as a priori, $\mathcal{P}_{1}$ is a deterministic convex optimization problem and thus easy to solve. However, in practice, the accurate measurement of probability distribution is a nontrivial task. In [26], we utilized a stochastic approximation-based approach to circumvent the difficulty of estimating the probability distribution. In the following, we will extend this technique and develop a tailored distributed algorithm to address the issues of time-varying link capacities as well as the user-specific QoS requirements, which are of particular interest in multihop cognitive wireless mesh networks.

First, define the Lagrangian function of $\mathcal{P}_{1}$ as

$$
\begin{aligned}
& L(\mathbf{r}, \lambda, \mu, \mathbf{v}) \\
& =\sum_{l \in \mathbb{L}} U_{l}\left(\sum_{k \in \mathbb{P}_{l}} r_{l}^{k}\right)+\sum_{l \in \mathbb{L}} \lambda_{l}\left(d_{l}-\sum_{k \in \mathbb{P}_{l}} r_{l}^{k}\right) \\
& +\sum_{l \in \mathbb{L}} v_{l}\left(b_{l}-\sum_{s \in \mathbb{S}} \pi_{s} \sum_{k \in \mathbb{P}_{l}} r_{l}^{k}\left(\sum_{e \in P_{l}^{k}} l_{e}^{s}\left(f_{e}, c_{e}^{s}\right)\right)\right) \\
& -\sum_{e \in \mathbb{E}} \mu_{e}\left(f_{e}-\sum_{s \in \mathbb{S}} \pi_{s} c_{e}^{s}\right) \\
& =\sum_{l \in \mathbb{L}}\left\{U_{l}\left(\sum_{k \in \mathbb{P}_{l}} r_{l}^{k}\right)+\lambda_{l}\left(d_{l}-\sum_{k \in \mathbb{P}_{l}} r_{l}^{k}\right)+v_{l} b_{l}\right. \\
& \left.-v_{l} \sum_{s \in \mathbb{S}} \pi_{s} \sum_{k \in \mathbb{P}_{l}} r_{l}^{k}\left(\sum_{e \in P_{l}^{k}} l_{e}^{s}\left(f_{e}, c_{e}^{s}\right)\right)\right\} \\
& -\sum_{e \in \mathbb{E}} \mu_{e}\left(f_{e}-\sum_{s \in \mathbb{S}} \pi_{s} c_{e}^{s}\right) \\
& =\sum_{s \in \mathbb{S}} \pi_{s}\left\{\sum _ { l \in \mathbb { L } } \left(U_{l}\left(\sum_{k \in \mathbb{P}_{l}} r_{l}^{k}\right)+\lambda_{l}\left(d_{l}-\sum_{k \in \mathbb{P}_{l}} r_{l}^{k}\right)+v_{l} b_{l}\right.\right. \\
& \left.-\sum_{k \in \mathbb{P}_{l}} r_{l}^{k}\left(\sum_{e \in P_{l}^{k}}\left(v_{l} l_{e}^{s}\left(f_{e}, c_{e}^{s}\right)+\mu_{e}\right)\right)+\sum_{e \in \mathbb{E}} \mu_{e} c_{e}^{s}\right\} \text {. }
\end{aligned}
$$

Define

$$
\begin{aligned}
\mathcal{M}^{s}(\lambda, \mu, v) & \\
= & \sup _{\mathbf{r} \geq 0}\left\{\sum _ { l \in \mathbb { L } } \left(U_{l}\left(\sum_{k \in \mathbb{P}_{l}} r_{l}^{k}\right)+\lambda_{l}\left(d_{l}-\sum_{k \in \mathbb{P}_{l}} r_{l}^{k}\right)+v_{l} b_{l}\right.\right. \\
& \left.\left.-\sum_{k \in \mathbb{P}_{l}} r_{l}^{k}\left(\sum_{e \in P_{l}^{k}}\left(v_{l} l_{e}^{s}\left(f_{e}, c_{e}^{s}\right)+\mu_{e}\right)\right)\right)+\sum_{e \in \mathbb{E}} \mu_{e} c_{e}^{s}\right\} .
\end{aligned}
$$

Let $\tilde{\mathbf{r}}$ be the optimum solution of (7). We will discuss how to obtain $\tilde{\mathbf{r}}$ shortly. The dual function of $\mathcal{P}_{1}$ is obtained by

$$
g(\lambda, \mu, v)=\sum_{s \in \mathbb{S}} \pi_{s} \mathcal{M}^{s}(\lambda, \mu, v) .
$$

Thus, the dual problem of $\mathcal{P}_{1}$ is given by

$$
\underline{\mathcal{P}_{2}:} \quad \min _{\lambda, \mu, \mathbf{v} \geq 0} g(\lambda, \mu, \mathbf{v}) .
$$

\subsection{Distributed Algorithmic Solution with the Stochastic Primal-Dual Approach}

In this section, we propose a distributed algorithmic solution of $\mathcal{P}_{1}$, or equivalently $\mathcal{P}_{2}$, based on the stochastic primal-dual method. In order to reach the stochastic optimum solution, the dual variables $\lambda, \mu$, and $\mathbf{v}$ are updated according to the following dynamics

$$
\begin{gathered}
\lambda_{l}(n+1)=\left[\lambda_{l}(n)-\alpha_{l}(n) \zeta_{l}(n)\right]^{+} \forall l \in \mathbb{L}, \\
\mu_{e}(n+1)=\left[\mu_{e}(n)-\alpha_{e}(n) \xi_{e}(n)\right]^{+} \forall e \in \mathbb{E}, \\
v_{l}(n+1)=\left[v_{l}(n)-\alpha_{b}(n) \rho_{l}(n)\right]^{+} \forall l \in \mathbb{L},
\end{gathered}
$$

where $[x]^{+}$denotes $\max (0, x)$ and $n$ is the iteration number. $\alpha_{l}(n), \alpha_{e}(n)$, and $\alpha_{b}(n)$ are the current step sizes while $\zeta_{l}(n)$, $\xi_{e}(n)$, and $\rho_{l}(n)$ are random variables. More precisely, they are named as the stochastic subgradient of the dual function $g(\lambda, \mu)$ and the following requirements need to be satisfied

$$
\begin{aligned}
& \mathrm{E}\left\{\zeta_{l}(n) \mid \lambda(1), \ldots, \lambda(n)\right\}=\partial_{\lambda_{l}} g(\lambda, \mu, \mathbf{v}) \forall l \in \mathbb{L}, \\
& \mathrm{E}\left\{\xi_{e}(n) \mid \mu(1), \ldots, \mu(n)\right\}=\partial_{\mu_{e}} g(\lambda, \mu, \mathbf{v}) \forall e \in \mathbb{E}, \\
& \mathrm{E}\left\{\rho_{l}(n) \mid \mathbf{v}(1), \ldots, \mathbf{v}(n)\right\}=\partial_{v_{l}} g(\lambda, \mu, \mathbf{v}) \forall l \in \mathbb{L},
\end{aligned}
$$

where $\mathrm{E}($.$) is the expectation operator and \lambda(1), \ldots, \lambda(n)$, $\mu(1), \ldots, \mu(n)$, and $\mathbf{v}(1), \ldots, \mathbf{v}(n)$ denote the sequences of solutions generated by (10), (11), and (12), respectively. By Danskin's Theorem [36], we can obtain the subgradients as

$$
\begin{gathered}
\zeta_{l}(n)=d_{l}-\sum_{k \in \mathbb{P}_{l}} \tilde{r}_{l}^{k}(n) \forall l \in \mathbb{L}, \\
\xi_{e}(n)=c_{e}^{s}(n)-\tilde{f}_{e}(n) \forall e \in \mathbb{E}, \\
\rho_{l}(n)=b_{l}-\sum_{k \in \mathbb{P}_{l}} \tilde{r}_{l}^{k}(n) \sum_{e \in P_{l}^{k}} l_{e}^{s}\left(\tilde{f}_{e}(n), c_{e}^{s}(n)\right) \forall l \in \mathbb{L},
\end{gathered}
$$

where $\tilde{r}_{l}^{k}$ is the optimum solution of (7). Note that $c_{e}^{s}(n)$ denotes the instantaneous channel capacity on link $e$ at iteration $n$.

We next show how to calculate $\mathcal{M}^{s}(\lambda, \mu, \mathbf{v})$ in (7), i.e., finding the optimum solution, denoted by $\tilde{\mathbf{r}}$, which maximizes

$$
\begin{aligned}
& \sum_{l \in \mathbb{L}}\left(U_{l}\left(\sum_{k \in \mathbb{P}_{l}} r_{l}^{k}\right)+\lambda_{l}\left(d_{l}-\sum_{k \in \mathbb{P}_{l}} r_{l}^{k}\right)+v_{l} b_{l}\right. \\
& \left.-\sum_{k \in \mathbb{P}_{l}} r_{l}^{k}\left(\sum_{e \in P_{l}^{k}}\left(v_{l} l_{e}^{s}\left(f_{e}, c_{e}^{s}\right)+\mu_{e}\right)\right)\right)+\sum_{e \in \mathbb{E}} \mu_{e} c_{e}^{s} .
\end{aligned}
$$

Note that when updating the primal variable, i.e., $\mathbf{r}$, the link costs are deterministic which are obtained via the feedback signal, e.g., ACK messages. Therefore, by utilizing the same stochastic subgradient approach, we have

$$
r_{l}^{k}(n+1)=\left[r_{l}^{k}(n)+\alpha_{r}(n) \eta(n)\right]_{0}^{d_{l}},
$$


where

$$
\eta(n)=\frac{\partial U_{l}}{\partial \sum_{k \in \mathbb{P}_{l}} r_{l}^{k}(n)}-\lambda_{l}-\sum_{e \in P_{l}^{k}}\left(\mu_{e}+v_{l} l_{e}\left(f_{e}, c_{e}^{s}\right)\right)
$$

is the stochastic subgradient measured at time $n$.

Theorem 1. The proposed algorithm converges to the global optimum of $\mathcal{P}_{1}$ with probability one, if the following constraints of step sizes are satisfied: 1) $\alpha(n)>0$, 2) $\sum_{n=0}^{\infty} \alpha(n)=\infty$, and 3) $\sum_{n=0}^{\infty}(\alpha(n))^{2}<\infty, \forall l \in \mathbb{L}$ and $e \in \mathbb{E}$, where $\alpha$ represents $\alpha_{e}, \alpha_{l}, \alpha_{b}$, and $\alpha_{r}$ generally.

Proof. First, let us revisit the updating equations of (10)-(12). Note that in the stochastic subgradient approach, the measured values of $\zeta_{l}(n), \xi_{e}(n)$, and $\rho_{l}(n)$ are considered as the instantaneous observation of the real gradients, denoted by $\overline{\zeta_{l}(n)}, \overline{\xi_{e}(n)}$, and $\overline{\rho_{l}(n)}$, respectively. We consider the relationship of $\zeta_{l}(n)$ and $\overline{\zeta_{l}(n)}$ for instance. The observation value, i.e., $\zeta_{l}(n)$, can be rewritten as

$$
\begin{aligned}
\zeta_{l}(n) & =\zeta_{l}(n)-\mathrm{E}\left(\zeta_{l}(n)\right)+\mathrm{E}\left(\zeta_{l}(n)\right)-\overline{\zeta_{l}(n)}+\overline{\zeta_{l}(n)} \\
& =\overline{\zeta_{l}(n)}+\mathrm{E}\left(\zeta_{l}(n)\right)-\overline{\zeta_{l}(n)}+\zeta_{l}(n)-\mathrm{E}\left(\zeta_{l}(n)\right) \\
& =\overline{\zeta_{l}(n)}+\widehat{\zeta_{l}(n)}+\widehat{\zeta_{l}(n)},
\end{aligned}
$$

where $\mathrm{E}$ is the expectation operator and

$$
\begin{gathered}
\widetilde{\zeta_{l}(n)}=\mathrm{E}\left(\zeta_{l}(n)\right)-\overline{\zeta_{l}(n)}, \\
\widehat{\zeta_{l}(n)}=\zeta_{l}(n)-\mathrm{E}\left(\zeta_{l}(n)\right) .
\end{gathered}
$$

Note that $\widetilde{\zeta_{l}(n)}$ is the difference between the expectation of the observations and the real gradient. Hence, it is the biased estimation error term. Next, we examine that

$$
\mathrm{E}\left(\zeta_{l} \widehat{(n)} \mid \zeta_{l}(\widehat{n-1}), \ldots, \widehat{\zeta_{l}(0)}\right)=0 \forall n .
$$

Therefore, the series of $\widehat{\zeta_{l} \widehat{(n)}}$ is a martingale difference sequence [37]. The relationship of (22) indicates that the observation value is the real gradient disturbed by a biased estimation error as well as a martingale difference noise. We next investigate the convergence conditions of the stochastic primal-dual approach. For $\overline{\zeta_{l}(n)}$, the following requirement

$$
\sum_{n=0}^{\infty} \alpha_{e}(n)\left|\mathrm{E}\left(\zeta_{l}(n)\right)-\overline{\zeta_{l}(n)}\right|<\infty
$$

is satisfied due to the stationary assumption. Similarly,

$$
\mathrm{E}\left(\zeta_{l} \widehat{(n)}^{2}\right)=\mathrm{E}\left(\left(\zeta_{l}(n)-\mathrm{E}\left(\zeta_{l}(n)\right)\right)^{2}\right)
$$

is bounded as well. The similar analysis can be extended to $\xi_{e}(n), \rho_{l}(n)$, and $\eta(n)$ in (11), (12), and (20) straightforwardly. Therefore, the standard conditions are satisfied and the convergence result of Theorem 1 follows the recent work of [38].

It is worth noting that the aforementioned distributed algorithm enjoys the merit of distributed implementation from an engineering perspective. With the current values of dual variables, each source node $S(l)$ optimizes (19) according to (21) and (20). The information needed is either locally attainable or acquirable by the feedback along the paths. For example, the channel states of the intermediate nodes along paths can be piggybacked by the end-to-end acknowledgement messages from the destination node, i.e., the gateway node in our scenario. The source node updates the $\lambda_{l}$ and $v_{l}$ according to (10) and (12) where the needed information is calculated by (16) and (18), respectively. For each link $e$, the current status of (17) is measured. Next, the value of $\mu_{e}$ is updated following (11). The iteration continues until an equilibrium point is reached. Note that our framework can incorporate the wireless lossy network scenarios by replacing the flow rate with the effective flow rate in the leaky-pipe flow model [39].

\section{Stochastic Traffic Engineering Without CONVEXITY}

Thus far, we have considered the scenarios where all the users have concave utility functions. However, in practice, several network applications may possess a nonconcave utility function. For example, in a data streaming application, the user is satisfied if the achieved data rate exceeds a threshold, where the utility function is a step function and thus the convexity does not preserve. Therefore, the proposed stochastic primal-dual approach in Section 4 cannot be applied here. It is worth noting that we can still formulate the stochastic traffic engineering problem as in $\mathcal{P}_{1}$ except that the optimization problem is a stochastic nonconvex programming, which is NP-hard in general, and computationally prohibitive to solve even in a centralized fashion [40]. In the following section, we will propose an algorithmic solution to the nonconvex stochastic traffic engineering problem, based on the learning automata techniques. Moreover, we analytically show that the proposed algorithm will converge to the global optimum solution asymptotically, in a decentralized fashion.

\subsection{Decentralized Algorithmic Solution with the Learning Automata Techniques}

We first convert the compact strategy space of each user into a discretized set denoted by $\mathbb{R}$. More specifically, each user, say $l$, maintains a probability vector $\mathbf{p}_{l, k}$ for each path $k \in \mathbb{P}_{l}$. The segment of $\left[0, r^{m}\right]$ is quantized into $Q$ sections where $r^{m}$ is the maximum allowed transmission rate on any path. In other words, the continuous variable $r_{l}^{k}$ is transformed into a discrete random variable, $r_{l, k}^{q}$, within a discretized set $\mathbb{R}$ with $Q+1$ elements. The data rate is randomly selected from $\mathbb{R}$ according to the probability vector of $\mathbf{p}_{l, k}$ where the $q$ th element, $p_{l, k}^{q}, q=0, \ldots, Q$, denotes the probability that the $l$ th user transmits with a rate of $r_{l, k}^{q}=q \times \frac{r^{m}}{Q}$ on the $k$ th path of $\mathbb{P}_{l}$. Associated with each probability vector $\mathbf{p}_{l, k}$, there is a weighting vector $\mathbf{w}_{l, k}$ with the same dimension of $1 \times(Q+1)$. The probability vector $\mathbf{p}_{l, k}$ is uniquely determined by the weighting vector $\mathbf{w}_{l, k}$ by the softmax function [41],

$$
p_{l, k}^{q}=\frac{e^{w_{l, k}^{q}}}{\sum_{q=0}^{Q} e^{w_{l, k}^{q}}} \forall l, k, q,
$$

where $w_{l, k}^{q}$ is the $q$ th element of $\mathbf{w}_{l, k}, q=0, \ldots, Q$. 
Next, we formulate an identical interest game where the players are the $|\mathbb{L}|$ source nodes and the common objective function is the overall network utility, i.e., the summation of the utility functions. In addition, for each source node, a team of learning automata [42] is constructed. At each time step, every source node picks the data rates on its own paths according to the probability vectors, which are determined by the weighting vectors. Based on the feedback signal $\Xi$, which will be defined shortly, each source node adjusts the weighting vectors and the iteration continues. The executed algorithm on every source node, say $l$, is provided as follows:

\section{Algorithm.}

\section{Repeat:}

- $\quad$ For every path, say $k$, randomly selects a transmission rate $r_{l, k}^{j}$ from $\mathbb{R}$, according to the current probability vector $\mathbf{p}_{l, k}(n)$ where $n$ denotes the current time slot.

- After receiving the feedback signal $\Xi(n)$ from the gateway node, if the cost constraint is satisfied, the weighting vector $\mathbf{w}_{l, k}$ is updated as

$$
\begin{aligned}
w_{l, k}^{q}(n+1)= & {\left[w_{l, k}^{q}(n)+\tau(n) \Xi(n)\left(1-\frac{e^{w_{l, k}^{q}}}{\sum_{q=0}^{Q} e^{w_{l, k}^{q}}}\right)\right.} \\
& \left.+\sqrt{\tau(n)} \varsigma_{l, k}^{q}(n)\right]_{0}^{\mathcal{L}}, \quad \text { for } q=j ; \\
w_{l, k}^{q}(n+1)= & {\left[w_{l, k}^{q}(n)+\sqrt{\tau(n)} \varsigma_{l, k}^{q}(n)\right]_{0}^{\mathcal{L}}, \quad \text { for } q \neq j . }
\end{aligned}
$$

Otherwise, the weighting vector remains the same.

- $\quad$ The probability vector $\mathbf{p}_{l, k}$ is then updated, following (28).

Until:

- $\max \left(\mathbf{p}_{l, k}(n+1)\right)>B$ where $B$ is a predefined convergence threshold.

In the algorithm, $\tau(n)$ is the learning parameter of the algorithm satisfying $0<\tau(n)<1$. $\mathcal{L}$ is a sufficiently large yet finite number which keeps the weighting vector bounded. The sequence of $\varsigma_{l, k}^{q}(n)$ is a set of i.i.d. random variables with zero mean and a variance of $\sigma^{2}(n)$. The global feedback signal $\Xi(n)$ is calculated by the gateway node and sent back to all source nodes, as

$$
\Xi(n)=\frac{\sum_{l \in \mathcal{L}}\left(U_{l}\left(\sum_{k \in \mathbb{P}_{l}} r_{l}^{k}\right)\right)}{\mathcal{J}},
$$

where $\mathcal{J}$ is a number to normalize the feedback signal. For example, we can set $\mathcal{J}$ to the maximum value of overall utility till $n$ and update this value on the fly. Therefore, the value of $\Xi(n)$ lies within $[0,1] . U_{l}$ is the nonconvex utility function for the $l$ th user. Note that the utility functions of all users are assumed to be truly acquired by the gateway node [43]. In practice, the value of $\Xi(n)$ can be circulated efficiently by established multicast algorithms such as [44]. Based on the feedback, the learning automata team adjusts the weighting vector in a decentralized fashion. In addition, note that $B$ is the predefined convergence threshold, e.g., $B=0.999$, which provides a tradeoff between the performance of the algorithm and its convergence speed.
Before analyzing the steady-state behavior of the proposed algorithm, we first discuss the following concepts:

Definition 1. Denote the maximum network utility of the original traffic engineering problem, i.e., $\mathcal{P}_{1}$, as $O^{*}$. Next, we define the final outcome of the proposed algorithm as $O^{\prime}$. We say that the algorithm provides an $\epsilon$-accurate solution, if for any arbitrarily small $\epsilon>0$, there exists a $Q^{\prime}$ such that

$$
\left|O^{*}-O^{\prime}\right|<\epsilon \forall Q>Q^{\prime}
$$

Definition 2. A potential game [45] is defined as a game where there exists a potential function $V$ such that

$$
V\left(a^{\prime}, a_{-l}\right)-V\left(a^{\prime \prime}, a_{-l}\right)=U_{l}\left(a^{\prime}, a_{-l}\right)-U_{l}\left(a^{\prime \prime}, a_{-l}\right) \forall l, a^{\prime}, a^{\prime \prime},
$$

where $U_{l}$ is the utility function for player $l$ and $a^{\prime}, a^{\prime \prime}$ are two arbitrary strategies in its strategy space. The notation of $a_{-l}$ denotes the vector of choices made by all players other than $l$.

Definition 3. A weighted potential game [45] is defined as a game where there exists a potential function $V$ such that

$$
\left(V\left(a^{\prime}, a_{-l}\right)-V\left(a^{\prime \prime}, a_{-l}\right)\right) \times h_{i}=U_{l}\left(a^{\prime}, a_{-l}\right)-U_{l}\left(a^{\prime \prime}, a_{-l}\right),
$$

for all $l, a^{\prime}, a^{\prime \prime}$ where $h_{i}>0$.

According to the definitions, it is apparent that the formulated identical interest game is a special case of weighted potential games. In the following theorem, we will provide the convergence behavior of a more general setting for weighted potential games, and hence the result applies to our specific scenario naturally:

Theorem 2. For an N-person weighted potential game where each person represents a team of learning automata, the proposed algorithm can converge to the global optimum solution asymptotically, which is an $\epsilon$-accurate solution to the original stochastic traffic engineering problem, for sufficient small value of $\tau$ and $\sigma$.

Proof. We defer the proof of Theorem 2 to Appendix.

Note that Theorem 2 establishes the convergence behavior of the aforementioned algorithm with no additional requirement for the problem structure. In contrast, we propose a stochastic primal-dual approach in Section 4, which requires the underlying problem to be a stochastic convex programming. The stochastic primal-dual approach cannot be applied efficiently otherwise. However, the aforementioned learning-based algorithm is suitable for almost every aspect such as stochastic nonconvex programming and stochastic mixed-integer programming. The asymptotically convergence result still holds. Therefore, in this paper, the proposed algorithms provide two different exemplifying methods for protocol designs under the stochastic environment. However, it is worth noting that for the latter approach, the tradeoff for general applicability is the convergence speed. In other words, in order to achieve an accurate result, i.e., when $\epsilon$ is small, the convergence speed may be slow. The actual convergence speed depends on the values of $\epsilon, Q, \tau, \sigma$ as well as the inherent structure of the problem and hence is difficult to 


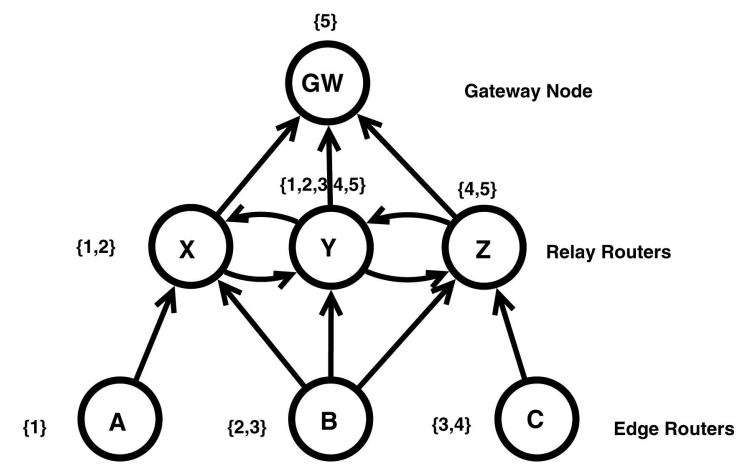

Fig. 2. Example of cognitive wireless mesh network.

quantify. Fortunately, in practical applications, achieving a "good enough" result is sometimes satisfactory. This tradeoff can be achieved by utilizing diminishing values of $\tau$ and $\sigma$, as demonstrated in the simulated annealing literature [46] and a recent work of [47]. To sum up, if the stochastic traffic engineering problem possesses a nice property of convexity, the algorithm based on the stochastic primaldual approach in Section 4 is recommended due to its nice decomposed structure and computationally efficient solution. However, if the problem is nonconvex in nature, the learning automata-based algorithm can be utilized to achieve an approximate solution. The tradeoff between the accuracy and convergence speed can be tuned by adjusting the values of $\tau$ and $\sigma$.

\section{Performance Evaluation}

In this section, we present a simple yet illustrative example to demonstrate the theoretical results.

We consider a cognitive wireless mesh network ${ }^{3}$ depicted in Fig. 2. There are three edge routers as the source nodes, denoted by $A, B$, and $C$, which transmit to the gateway node $G W$ via the relay routers $X, Y$, and $Z$. Among all feasible paths, we select the following available paths for edge routers, as summarized in Table 1.

There are five primary users in the area, denoted by 1-5 where each one has a primary band of $10 \mathrm{MHz}$. The common ISM band is assumed to be $10 \mathrm{MHz}$. The return probability of the primary users is given as $\varpi=[0.2,0.3$, $0.4,0.3,0.3]$. The transmitting power of each node is fixed as $100 \mathrm{~mW}$, and the noise power is assumed to be $3 \mathrm{~mW}$. We consider a model where the received power is inversely proportional to the square of the distance. Note that the transmitting power is uniformly spread on all available bands. In addition, we explicitly specify the affecting primary users for a particular node. We use $\{i, j, k, \ldots\}$ to represent that a particular node is affected by primary user $i, j, k, \ldots$. For example, node $X$ is labeled with $\{1,2\}$ which indicates that the transmission of node $X$ will devastate the transmissions of primary user 1 and 2 if the corresponding primary band is utilized. Note that the central node, namely, $Y$, is most severely affected by all primary users. Intuitively, to achieve an expected optimum solution, the

3. Fig. 2 only shows the links on the available paths obtained by the signaling mechanisms or manual configurations. The actual physical topology of the network can be potentially larger.
TABLE 1

Available Paths for Edge Routers

\begin{tabular}{||c|c|c||}
\hline \multirow{4}{*}{$A$} & $P_{A}^{1}:$ & $\{A \rightarrow X \rightarrow G W\}$ \\
\cline { 2 - 3 } & $P_{A}^{2}:$ & $\{A \rightarrow X \rightarrow Y \rightarrow G W\}$ \\
\cline { 2 - 3 } & $P_{A}^{3}:$ & $\{A \rightarrow X \rightarrow Y \rightarrow Z \rightarrow G W\}$ \\
\hline \multirow{4}{*}{$B$} & $P_{B}^{1}:$ & $\{B \rightarrow X \rightarrow G W\}$ \\
\cline { 2 - 3 } & $P_{B}^{2}:$ & $\{B \rightarrow Y \rightarrow G W\}$ \\
\cline { 2 - 3 } & $P_{B}^{3}:$ & $\{B \rightarrow Y \rightarrow X \rightarrow G W\}$ \\
\cline { 2 - 3 } & $P_{B}^{4}:$ & $\{B \rightarrow Y \rightarrow Z \rightarrow G W\}$ \\
\cline { 2 - 3 } & $P_{B}^{5}:$ & $\{B \rightarrow Z \rightarrow G W\}$ \\
\hline \multirow{3}{*}{$C$} & $P_{C}^{1}:$ & $\{C \rightarrow Z \rightarrow G W\}$ \\
\cline { 2 - 3 } & $P_{C}^{2}:$ & $\{C \rightarrow Z \rightarrow Y \rightarrow G W\}$ \\
\cline { 2 - 3 } & $P_{C}^{3}:$ & $\{C \rightarrow Z \rightarrow Y \rightarrow X \rightarrow G W\}$ \\
\hline
\end{tabular}

stochastic traffic engineering algorithms are inclined to steer the traffic away from $Y$. We will demonstrate this detour effect next.

We first consider the cognitive wireless mesh network with convexity, e.g., $U_{l}\left(x_{l}\right)=\log x_{l}$ to achieve a proportional fairness among the flows [33]. The link cost is assumed to be in the form of $l_{e}^{s}\left(f_{e}, C_{e}^{s}\right)=\frac{1}{c_{e}^{s}-f_{e}}$, which reflects the delay experienced for a unit flow on link $e$ under the $M / M / 1$ assumption [48]. Note that if $f_{e} \geq c_{e}$, the cost is $+\infty$. We set the traffic demand of all edge routers as $d_{l}=30 \mathrm{Mbps}$ while the cost budget is $b_{l}=5$. The step sizes are chosen as $\alpha=$ $1 / n$ where $n$ is the current iteration step. Fig. 3a illustrates the trajectories of the rate variables, and Fig. $3 \mathrm{~b}$ shows the convergence of the network overall utility as well as the individual utility functions. ${ }^{4}$ We observe that while the rate variables converge as the iterations go, the overall objective, i.e., the sum of the individual utilities, approaches to the global optimum indicated by the dashed line, which is attained by calculating the steady-state distribution following the return probability $\varpi$.

In addition, Table 2 provides the rate on each path after convergence for a sample run of the algorithm. For comparison, we provide the convergence rates when node $Y$ is switched from the most affected node to the least affected node, i.e., node $Y$ can utilize all five primary bands all the time, in Table 3. From Tables 2 and 3, it is interesting to note that, in the first scenario, each user allocates a relatively small amount of flow on the paths which traverse through node $Y$. On the contrary, when node $Y$ is less affected, all the flows allocate noticeably larger data rates on paths that traverse through $Y$ despite the fact that node $Y$ is the central node which is least favorable by traditional traffic engineering solutions. Therefore, our proposed stochastic traffic engineering algorithm is of particular interest for multihop cognitive wireless mesh networks due to the capability of steering the traffic away from the severely affected areas automatically, without a prior knowledge of the underlying probabilistic structure, in a distributed fashion.

We next consider a cognitive wireless mesh network with nonconvex utility functions. Specifically, we consider the utility function as

$$
U_{l}\left(x_{l}\right)= \begin{cases}1, & \text { if } x_{l} \geq 2 \text { Mbps } \\ 0, & \text { if } x_{l}<2 \text { Mbps. }\end{cases}
$$

4. Note that Fig. $3 b$ also reflects the evolution of the throughput of each edge router logarithmically. 


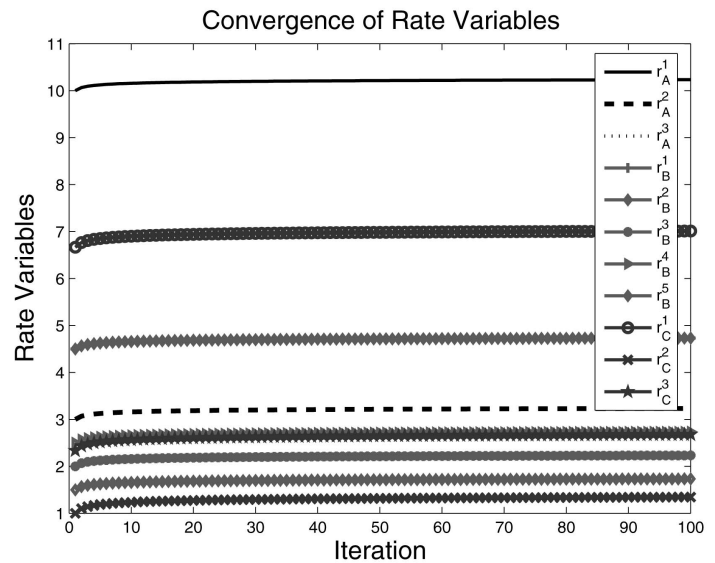

(a)

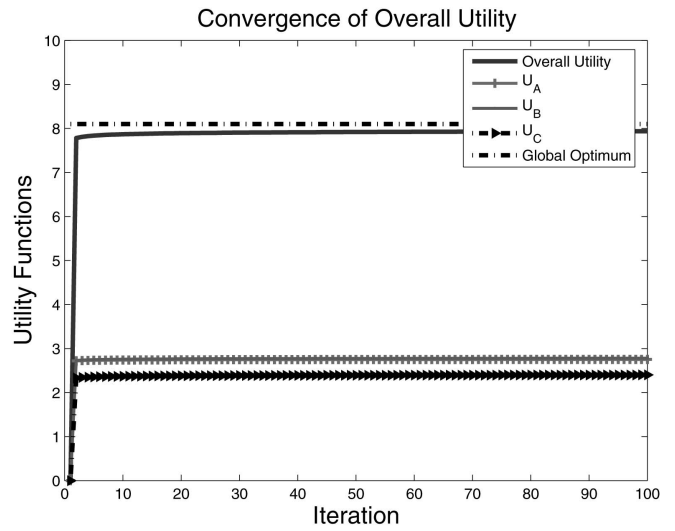

(b)

Fig. 3. Cognitive wireless mesh networks with convexity. (a) Trajectories of rate variables. (b) Trajectories of utility functions.

TABLE 2

Convergence Rates when $Y$ is Affected by All Five Primary Users

\begin{tabular}{||c|c|c||}
\hline$A$ & $P_{A}^{1}:$ & 15.3035 \\
\cline { 2 - 3 } & $P_{A}^{2}:$ & 3.2458 \\
\cline { 2 - 3 } & $P_{A}^{3}:$ & 2.7362 \\
\hline \multirow{4}{*}{$B$} & $P_{B}^{1}:$ & 7.3725 \\
\cline { 2 - 3 } & $P_{B}^{2}:$ & 1.9584 \\
\cline { 2 - 3 } & $P_{B}^{3}:$ & 2.1752 \\
\cline { 2 - 3 } & $P_{B}^{4}:$ & 2.6458 \\
\cline { 2 - 3 } & $P_{B}^{3}:$ & 5.3489 \\
\hline \multirow{4}{*}{$C$} & $P_{C}^{1}:$ & 17.3051 \\
\cline { 2 - 3 } & $P_{C}^{2}:$ & 2.3792 \\
\cline { 2 - 3 } & $P_{C}^{3}:$ & 2.5675 \\
\hline
\end{tabular}

while other settings are the same as in the previous scenario. Additionally, we utilize diminishing values of $\tau$ and $\sigma$ as $\tau=1 / n$ and $\sigma=1 / n$, where $n$ is the iteration step. ${ }^{5}$ Without the loss of generality, we set $\mathcal{L}=100$ and the quantization level $Q=20$. The maximum allowed rate $r^{m}$ is assumed to be 10. Therefore, the discretized data rate set is given by $\mathbb{R}=[0.5,1.0,1.5, \ldots, 9.0,9.5,10.0]$. Fig. 4 illustrates the evolution of the probability vector of $\mathbf{p}_{A, 1}$. Note that as the iterations evolve, the probability of $p_{A, 1}^{20}$, i.e., the probability

5. By utilizing diminishing parameters, a tradeoff between the performance and the convergence speed can be achieved by tuning the decreasing speed [46].
TABLE 3

Convergence Rates when $Y$ Is Not Affected by Any of the Primary Users

\begin{tabular}{||c|c|c||}
\hline \multirow{4}{*}{$A$} & $P_{A}^{1}:$ & 9.6413 \\
\cline { 2 - 3 } & $P_{A}^{2}:$ & 11.1335 \\
\cline { 2 - 3 } & $P_{A}^{3}:$ & 9.1861 \\
\hline \multirow{4}{*}{$B$} & $P_{B}^{1}:$ & 5.0101 \\
\cline { 2 - 3 } & $P_{B}^{2}:$ & 6.2625 \\
\cline { 2 - 3 } & $P_{B}^{3}:$ & 4.9711 \\
\cline { 2 - 3 } & $P_{B}^{4}:$ & 4.9921 \\
\cline { 2 - 3 } & $P_{B}^{5}:$ & 8.7878 \\
\hline \multirow{4}{*}{$C$} & $P_{C}^{1}:$ & 15.3118 \\
\cline { 2 - 3 } & $P_{C}^{2}:$ & 9.1042 \\
\cline { 2 - 3 } & $P_{C}^{3}:$ & 5.6121 \\
\hline
\end{tabular}

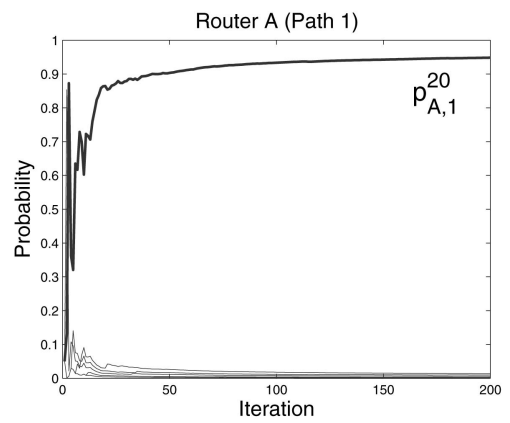

Fig. 4. Trajectory of the probability vector of router A's first path.

that router A chooses the 20th data rate ( $r^{m}$ in this case), excels others and approaches to 1 asymptotically. We plot the evolutions of the probability vectors of other paths in Fig. 5 collectively. For each path, the probability of selecting one particular data rate soon excels others. We observe that router A selects the twentieth, the first, and the fourth date rate on its three paths asymptotically. Meanwhile, router B inclines to choose the eighth, the second, the first, the fourth, and the twentieth data rate on its paths. The steady-state data rates for router $C$ is the twentieth, the first, and the first element in $\mathbb{R}$, as depicted in Fig. 5. It is interesting to notice that all the routers automatically detour the traffic from the severely affected node $Y$ by allocating more data rate on other paths.

\section{Conclusions}

In this paper, we investigate the STE problem in cognitive wireless mesh networks. To harness the randomness induced by the unpredictable behaviors of primary users, we formulate the STE problem in a stochastic network utility maximization framework. For the cases where convexity holds, we derive a distributed algorithmic solution via the stochastic primal-dual approach, which provably converges to the global optimum solution. For the scenarios where convexity is not attainable, we propose an alternative decentralized algorithmic solution based on the learning automata techniques. We show that the algorithm converges to the global optimum solution asymptotically, under certain conditions.

In our work, we restrict ourself in a single gateway scenario. The extension to the multiple gateway scenario seems interesting and needs further investigation. In addition, in this paper, we consider a cooperative case where all 

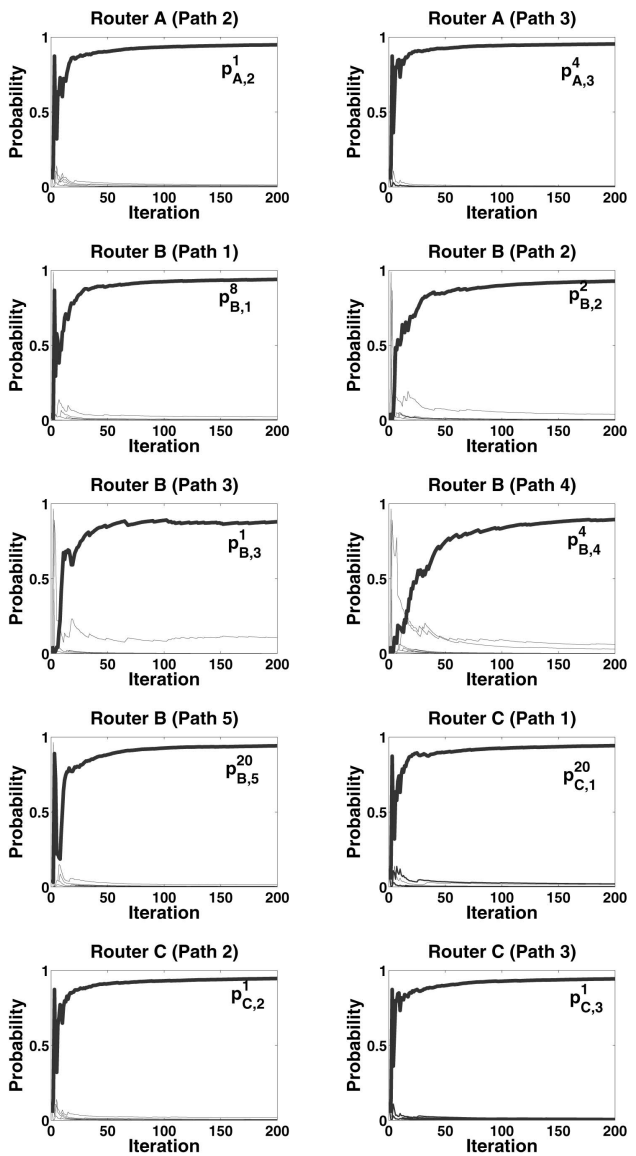

Fig. 5. Trajectories of the probability vectors on other paths.

the edge routers attempt to maximize the overall network performance. In the cases where the edge routers are noncooperative, each player is interested in its own utility rather than the social welfare. Stochastic game theory provides a feasible tool to address the noncooperative case, which remains as future research. We also assume a negligible delay for the feedback signal while in a more general case, the impact of feedback delay needs further investigation. One feasible solution is to utilize the distributed robust optimization framework [49] where the worst case performance is maximized given that the feedback delay/error is within a reasonable range. Our work initiates a first step to investigate the impact of unpredictable returns of primary users, on the stochastic traffic engineering problem in cognitive wireless mesh networks.

\section{APPENDIX}

\section{Proof of Theorem 2}

The proof follows similar lines as in [42]. However, we extend the result to a more general setting where the underlying $\mathrm{N}$-person stochastic game is a weighted potential game. Therefore, the proof in [42] can be viewed as a special case. Define $V$ as the potential function of the game. Note that the selected rate is determined by the probability vector which is generated uniquely by the weighting vector. Therefore, we can view the weighting vector as the variable in this case and the objective function is given by
$z=\mathrm{E}(V \mid \mathbf{w})$. In the updating procedure of (29), signal $\Xi$ is replaced by $V$. We first verify that

$$
\begin{aligned}
\frac{\partial z}{\partial w_{l, k}^{q}} & =\frac{\partial \mathrm{E}(V \mid \mathbf{w})}{\partial w_{l, k}^{q}} \\
& =\frac{\partial \sum_{q} p_{l, k}^{q} \mathrm{E}\left(V \mid \mathbf{w}, r_{l, k}^{q}\right)}{\partial w_{l, k}^{q}} \\
& =\sum_{q} \frac{e^{w_{l, k}^{q}}}{\sum_{q=0}^{Q} e^{w_{l, k}^{q}}}\left(1-\frac{e^{u_{l, k}^{q}}}{\sum_{q=0}^{Q} e^{w_{l, k}^{q}}}\right) \mathrm{E}\left(V \mid \mathbf{w}(n), r_{l, k}^{q}\right) .
\end{aligned}
$$

Note that

$$
\begin{aligned}
& \mathrm{E}\left(V\left(1-\frac{e^{w_{l, k}^{q}}}{\sum_{q=0}^{Q} e^{w_{l, k}^{q}}}\right) \mid \mathbf{w}(n)\right) \\
& =\sum_{q} p_{l, k}^{q}\left(1-\frac{e^{w_{l, k}^{q}}}{\sum_{q=0}^{Q} e^{w_{l, k}^{q}}}\right) \mathrm{E}\left(V \mid \mathbf{w}(n), r_{l, k}^{q}\right) \\
& =\sum_{q} \frac{e^{w_{l, k}^{q}}}{\sum_{q=0}^{Q} e^{w_{l, k}^{q}}}\left(1-\frac{e^{w_{l, k}^{q}}}{\sum_{q=0}^{Q} e^{w_{l, k}^{q}}}\right) \mathrm{E}\left(V \mid \mathbf{w}(n), r_{l, k}^{q}\right) \\
& =\frac{\partial z}{\partial w_{l, k}^{q}} .
\end{aligned}
$$

Next, it is straightforward to verify that the standard conditions in [50, Chapter 6, Theorem 7] are satisfied. We omit the verifications since they are the similar procedures as in [42]. Thus, we conclude that the above dynamic weakly converges to the following SDE [50], [51]:

$$
d \mathbf{w}=\nabla z+\sigma d \mathcal{W},
$$

for a sufficiently small $\tau \rightarrow 0$ where $\sigma$ is the standard deviation of the i.i.d. random variables $\varsigma_{l, k}^{q}$ and $\mathcal{W}$ is a standard Wiener Process. Note that the SDE (35) falls into the category of Langevin equation [52] which is well known that the probability measure concentrates on the global maximum solution of $z$ for a sufficiently small $\sigma$ [42], [52]. Therefore, we conclude that in the weighted potential game scenario, the proposed algorithm will converge to the global optimum of the objective function, for the quantized data rate setting. The association of the $\epsilon$-accurate solution to the original stochastic traffic engineering problem of $\mathcal{P}_{1}$ follows the result of [53].

\section{ACKNOWLEDGMENTS}

This work was supported in part by the US National Science Foundation under Grant DBI-0529012 and Grant CNS0626881. This project was also partially supported by the 111 project under grant B08038 with Xidian University, Xi'an, China.

\section{REFERENCES}

[1] I.F. Akyildiz, W.Y. Lee, M.C. Vuran, and S. Mohanty, "Next Generation/Dynamic Spectrum Access/Cognitive Radio Wireless Networks: A Survey," Computer Networks J., vol. 50, pp. 2127-2159, Sept. 2006.

[2] K. Xing, X. Cheng, L. Ma, and Q. Liang, "Superimposed Code Based Channel Assignment in Multi-Radio Multi-Channel Wireless Mesh Networks," Proc. MobiCom, pp. 15-26, 2007. 
[3] H.T. Cheng, H. Jiang, and W. Zhuang, "Distributed Medium Access Control for Wireless Mesh Networks," Wireless Comm. and Mobile Computing, vol. 6, pp. 845-864, 2006.

[4] http://www.sharedspectrum.com, 2009.

[5] C. Ghosh and D.P. Agrawal, "Channel Assignment with Route Discovery (CARD) Using Cognitive Radio in Multi-Channel Multi-Radio Wireless Mesh Networks," Proc. Third Ann. IEEE Comm. Society Conf. Sensor, Mesh and Ad Hoc Comm. and Networks (SECON '06), 2006.

[6] T. Chen, H. Zhang, G.M. Maggio, and I. Chlamtac, "Cogmesh: A Cluster-Based Cognitive Radio Network," Proc. IEEE Symp. New Frontiers in Dynamic Spectrum Access Networks (DySPAN '07), 2007.

[7] A. AL-Fuquha, B. Khan, A. Rayes, M. Guizani, O. Awwad, and G.B. Brahim, "Opportunistic Channel Selection Strategy for Better QoS in Cooperative Networks with Cognitive Radio Capabilities," IEEE J. Selected Areas in Comm., vol. 26, no. 1, pp. 156-167, Jan. 2008.

[8] K.R. Chowdhury and I.F. Akyildiz, "Cognitive Wireless Mesh Networks with Dynamic Spectrum Access," IEEE J. Selected Areas in Comm., vol. 26, no. 1, pp. 168-181, Jan. 2008.

[9] http://wirelessman.org/le/, 2009.

[10] Y. Xi and E.M. Yeh, "Distributed Algorithms for Spectrum Allocation, Power Control, Routing, and Congestion Control in Wireless Networks," Proc. ACM MobiHoc, 2007.

[11] "Cognitive Wireless Mesh Network Project," http://www.ece. gatech.edu/research/labs/bwn/mesh, 2009.

[12] D. Awduche, L. Berger, D. Gan, T. Li, V. Srinivasan, and G. Swallow, "RSVP-TE: Extensions to RSVP for LSP Tunnels," Internet Draft, RFC 3209, 2001.

[13] B. Jamoussi, L. Andersson, R. Callon, R. Dantu, L. Wu, P. Doolan, T. Worster, N. Feldman, A. Fredette, M. Girish, E. Gray, J. Heinanen, T. Kilty, and A. Malis, "Constraint-Based LAP Setup Using LDP," Internet Draft, RFC 3212, 2002.

[14] D. Mitra and Q. Wang, "Stochastic Traffic Engineering for Demand Uncertainty and Risk-Aware Network Revenue Management," IEEE/ACM Trans. Networking, vol. 13, no. 2, pp. 221-233, Apr. 2005.

[15] H. Wang, H. Xie, L. Qiu, Y.R. Yang, Y. Zhang, and A. Greenberg, "Cope: Traffic Engineering in Dynamic Networks," Proc. ACM Sigcomm, 2006.

[16] A. Elwalid, C. Jin, S. Low, and I. Widjaja, "Mate: MPLS Adaptive Traffic Engineering," Proc. IEEE INFOCOM, pp. 1300-1309, 2001.

[17] J. He, M. Bresler, M. Chiang, and J. Rexford, "Towards Robust Multi-Layer Traffic Engineering: Optimization of Congestion Control and Routing," IEEE J. Selected Areas in Comm., vol. 25, no. 5, pp. 868-880, June 2007.

[18] Q. Wang and H. Zheng, "Route and Spectrum Selection in Dynamic Spectrum Networks," Proc. IEEE Symp. New Frontiers in Dynamic Spectrum Access Networks (DySPAN '05), 2005.

[19] C. Xin, B. Xie, and C.-C. Shen, "A Novel Layered Graph Model for Topology Formation and Routing in Dynamic Spectrum Access Networks," Proc. IEEE Symp. New Frontiers in Dynamic Spectrum Access Networks (DySPAN '05), 2005.

[20] Y. Shi and T. Hou, "Optimal Power Control for Programmable Radio Networks," Proc. IEEE INFOCOM, 2007.

[21] T. Hou, Y. Shi, and H. Sherali, "Optimal Spectrum Sharing for Multi-Hop Software Defined Radio Networks," Proc. IEEE INFOCOM, 2007.

[22] T. Hou, Y. Shi, and H. Sherali, "Spectrum Sharing for Multi-Hop Networking with Cognitive Radios," IEEE J. Selected Areas in Comm., vol. 26, no. 1, pp. 146-155, Jan. 2008

[23] Y. Shi and T. Hou, "A Distributed Optimization Algorithm for Multi-Hop Cognitive Radio Networks," Proc. IEEE INFOCOM, 2008.

[24] J.-W. Lee, R.R. Mazumdar, and N.B. Shroff, "Opportunistic Power Scheduling for Dynamic Multi-Server Wireless Systems," IEEE Trans. Wireless Comm., vol. 5, no. 6, pp. 1506-1515, June 2006.

[25] J.-W. Lee, R.R. Mazumdar, and N.B. Shroff, "Joint Opportunistic Power Scheduling and End-to-End Rate Control for Wireless Ad-Hoc Networks," IEEE Trans. Vehicular Technology, vol. 56, no. 2, pp. 801-809, Mar. 2007.

[26] Y. Song, C. Zhang, and Y. Fang, "Routing Optimization in Wireless Mesh Networks under Uncertain Traffic Demands," Proc. Int'l Conf. Quality of Service in Heterogeneous Wired/Wireless Networks (QShine '08), 2008.

[27] A.A. Abidi, "The Path to the Software-Defined Radio Receiver," IEEE J. Solid-State Circuits, vol. 42, no. 5, pp. 954-966, May 2007.
[28] P.G. Cook and W. Bonser, "Architectural Overview of the Speakeasy System," IEEE J. Selected Areas in Comm., vol. 17, no. 4, pp. 650-661, Apr. 1999.

[29] F.K. Jondral, "Software-Defined Radio-Basics and Evolution to Cognitive Radio," EURASIP J. Wireless Comm. and Networking, vol. 3, pp. 275-283, 2005.

[30] H. Kim and K.G. Shin, "In-Band Spectrum Sensing in Cognitive Radio Networks: Energy Detection or Feature Detection?" Proc. ACM MobiCom, 2008.

[31] P. Kyasanur, J. So, C. Chereddi, and N.H. Vaidya, "Multi-Channel Mesh Networks: Challenges and Protocols," IEEE Wireless Comm., vol. 13, no. 2, pp. 30-36, Apr. 2006.

[32] A.R.S. Bahai, B.R. Saltzberg, and M. Ergen, Multi-carrier Digital Communications: Theory and Applications of OFDM. Springer, 2004.

[33] M. Chiang, "To Layer or Not to Layer: Balancing Transport and Physical Layers in Wireless Multihop Networks," Proc. IEEE INFOCOM, 2004.

[34] M. Chiang, S.H. Low, A.R. Calderbank, and J.C. Doyle, "Layering as Optimization Decomposition: A Mathematical Theory of Network Architectures," Proc. IEEE, vol. 95, no. 1, pp. 255-312, Mar. 2007.

[35] F. Kelly, A. Maulloo, and D. Tan, "Rate Control in Communication Networks: Shadow Prices, Proportional Fairness and Stability," J. Operation Research Soc., vol. 49, pp. 237-252, 1998.

[36] D.P. Bertsekas, Nonlinear Programming. Athena Scientific, 1999.

[37] H.J. Kushner and G.G. Yin, Stochastic Approximation and Recursive Algorithms and Applications. Springer, 2003.

[38] J. Zhang, D. Zheng, and M. Chiang, "The Impact of Stochastic Noisy Feedback on Distributed Network Utility Maximization," IEEE Trans. Information Theory, vol. 54, no. 2, pp. 645-665, Feb. 2008.

[39] Q. Gao, J. Zhang, and S.V. Hanly, "Cross-Layer Rate Control in Wireless Networks with Lossy Links: Leaky-Pipe Flow, Effective Network Utility Maximization and Hop-by-Hop Algorithms," Proc. IEEE INFOCOM, 2008.

[40] M.H. van der Vlerk, "Stochastic Programming Bibliography," World Wide Web, http:/ /mally.eco.rug.nl/spbib.html, 1996-2007.

[41] K. Swingler, Applying Neural Networks: A Practical Guide. Morgan Kaufmann, 1996.

[42] M.A.L. Thathachar and P.S. Sastry, Networks of Learning Automata. Kluwer Academic Publishers, 2004.

[43] D. Lehmann, L.I. Ocallaghan, and Y. Shoham, "Truth Revelation in Approximately Efficient Combinatorial Auctions," J. ACM, vol. 49, pp. 577-602, 2002.

[44] G. Zeng, B. Wang, Y. Ding, L. Xiao, and M. Mutka, "Multicast Algorithms for Multi-Channel Wireless Mesh Networks," Proc. IEEE Int'l Conf. Network Protocols (ICNP '07), 2007.

[45] D. Monderer and L. Shapley, "Potential Games," J. Games and Economic Behavior, vol. 14, pp. 124-143, 1996.

[46] P.J.M. van Laarhoven and E.H.L. Aarts, Simulated Annealing: Theory and Applications. Reidel, 1987.

[47] Y. Song, C. Zhang, and Y. Fang, "Throughput Maximization in Multi-Channel Wireless Mesh Access Networks," Proc. IEEE Int'l Conf. Network Protocols (ICNP '07), 2007.

[48] D. Bertsekas and R. Gallager, Data Networks, second ed. Prentice Hall, 1991.

[49] K. Yang, Y. Wu, J. Huang, X. Wang, and S. Verdu, "Distributed Robust Optimization for Communication Networks," Proc. IEEE INFOCOM, 2008.

[50] H.J. Kushner, Approximation and Weak Convergence Methods for Random Processes. MIT Press, 1984.

[51] P. Billingsley, Convergence of Probability Measures. Wiley, 1968.

[52] F. Aluffi-Pentini, V. Parisi, and F. Zirilli, "Global Optimization and Stochastic Difference Equations," J. Optimization Theory and Applications, vol. 47, pp. 1-26, 1985.

[53] A.S. Poznyak and K. Najim, Learning Automata and Stochastic Optimization. Springer, 1997. 


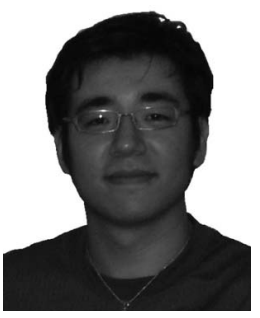

Yang Song received the BE and ME degrees in electrical engineering from Dalian University of Technology, China, and the University of Hawaii at Manoa, Honolulu, in July 2004 and August 2006, respectively. Since September 2006, he has been working toward the $\mathrm{PhD}$ degree in the Department of Electrical and Computer Engineering at the University of Florida, Gainesville. His research interests are wireless network, game theory, optimization, and mechanism design. He is a student member of the IEEE and a member of the Game Theory Society.

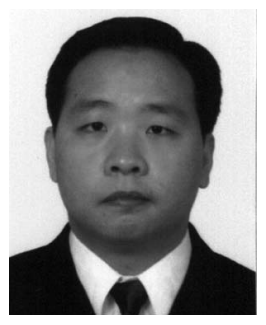

Chi Zhang received the $\mathrm{BE}$ and $\mathrm{ME}$ degrees in electrical engineering from Huazhong University of Science and Technology, Wuhan, China, in July 1999 and January 2002, respectively. Since September 2004, he has been working toward the $\mathrm{PhD}$ degree in the Department of Electrical and Computer Engineering at the University of Florida, Gainesville. His research interests are network and distributed system security, wireless networking, and mobile computing, with emphasis on mobile ad hoc networks, wireless sensor networks, wireless mesh networks, and heterogeneous wired/wireless networks.

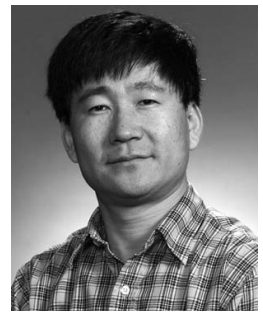

Yuguang Fang received the $\mathrm{PhD}$ degree in systems engineering from the Case Western Reserve University in January 1994 and the PhD degree in electrical engineering from Boston University in May 1997. He was an assistant professor in the Department of Electrical and Computer Engineering at New Jersey Institute of Technology from July 1998 to May 2000. He then joined the Department of Electrical and Computer Engineering at the University of Florida in May 2000 as an assistant professor, and got an early promotion to an associate professor with tenure in August 2003 and to a full professor in August 2005. He holds the University of Florida Research Foundation (UFRF) Professorship from 2006 to 2009 and the Changjiang Scholar Chair Professorship with the National Key Laboratory of Integrated Services Networks, Xidian University, China, from 2008 to 2011 . He has published more than 200 papers in refereed professional journals and conferences. He received the National Science Foundation Faculty Early Career Award in 2001 and the Office of Naval Research Young Investigator Award in 2002. He is the recipient of the Best Paper Award in the IEEE International Conference on Network Protocols (ICNP) in 2006 and the recipient of the IEEE TCGN Best Paper Award in the IEEE High-Speed Networks Symposium, IEEE Globecom, in 2002. He is also active in professional activities. He has served on several editorial boards of technical journals including the IEEE Transactions on Communications, the IEEE Transactions on Wireless Communications, the IEEE Transactions on Mobile Computing, and the ACM Wireless Networks. He has been actively participating in professional conference organizations such as serving as the steering committee cochair for QShine, the technical program vicechair for the IEEE INFOCOM'2005, the technical program symposium cochair for the IEEE Globecom'2004, and a member of the technical program committee for the IEEE INFOCOM $(1998,2000,2003-2009)$. He is a fellow of the IEEE and a member of the ACM.

$\triangleright$ For more information on this or any other computing topic, please visit our Digital Library at www.computer.org/publications/dlib. 